\title{
Suppressing Intersample Behavior in Iterative Learning Control
}

\author{
Tom Oomen, Jeroen van de Wijdeven, and Okko Bosgra
}

\begin{abstract}
Iterative Learning Control (ILC) is a control strategy to improve the performance of digital batch repetitive processes. Due to its digital implementation, discrete time ILC approaches do not guarantee good intersample behavior. In fact, common discrete time ILC approaches may deteriorate the intersample behavior, thereby reducing the performance of the sampled-data system. In this paper, a generally applicable multirate ILC approach is presented that enables to balance the at-sample performance and the intersample behavior. Furthermore, key theoretical issues regarding multirate systems are addressed, including the time-varying nature of the multirate ILC setup. The proposed multirate ILC approach is shown to outperform discrete time ILC in a realistic simulation example.
\end{abstract}

\section{INTRODUCTION}

Good at-sample performance is a necessary, yet not sufficient condition for good continuous time performance in sampled-data systems. Sampled-data systems include many physical systems that evolve in continuous time and are controlled by a digital controller [1]. Since the plant evolves in continuous time, it is most natural to evaluate performance in continuous time, i.e., to analyze both the at-sample response and the intersample behavior. In fact, achieving a good at-sample performance can go at the expense of a poor intersample behavior [2]. In this perspective, any high performance digital control design approach for a sampled-data system should be accompanied by a thorough intersample behavior consideration.

Iterative Learning Control (ILC) [3], [4], [5], [6] is a high performance digital control strategy used to improve the performance of batch repetitive processes, by iteratively updating the command signal from one experiment (trial) to the next. Basically, ILC results in a command signal that can compensate for trial-invariant deterministic components in the discretized error signal, even if imperfect plant knowledge is available.

Although ILC for discrete time LTI systems based on discretized error signals has been well developed, a systematic procedure to analyze and possibly improve the intersample behavior in ILC is lacking. In this paper, ILC for sampleddata systems is investigated.

Control design for sampled-data systems [1], [7] includes the following aspects that are not present in control design for continuous time systems:

- sampling zeros [8], [9], [1];

- aliased poles [10], [1]; and

- aliased disturbances [2].

The authors are with the Eindhoven University of Technology, Eindhoven, The Netherlands, t.a.e.oomen@tue.nl, j.j.m.v.d.wijdeven@tue.nl, o.h.bosgra@tue.nl. This research is supported by Philips Applied Technologies, Eindhoven, The Netherlands.
These aspects should be appropriately dealt with in sampleddata control design, including ILC for sampled-data systems. ILC can attenuate disturbances up to the Nyquist frequency, i.e., there is no Bode sensitivity integral as in the attenuation of disturbances by feedback control [11], thereby potentially increasing undesirable intersample behavior.

In [12], [13], it is shown that sampling zeros [8] can indeed deteriorate the intersample response in ILC and $a d$ hoc solutions are provided for performance improvement. In [14], repetitive control, which is closely related to ILC, for sampled-data systems is considered. The proposed solutions to handle the intersample behavior require modifications to the sampler and hold function. However, these functions are commonly unalterable in practice. In [15], [16] repetitive control of sampled-data systems is considered by employing lifted system descriptions. Although intersample behavior is addressed in the approach, these methods do not directly extrapolate to ILC due to its batch repetitive behavior.

The main contribution of this paper is a systematic procedure that extends existing ILC approaches for sampled-data systems by explicitly dealing with the intersample behavior. By employing norm-optimal ILC [17], the intersample response is explicitly quantified in the optimization problem. In addition, a multirate ILC approach is pursued, where the measurement of fast sampled signals is fully exploited to generate a command signal at a low sampling frequency. Although multirate ILC has been considered before in [18], the intersample behavior has not been explicitly dealt with. The resulting multirate ILC problem is time-varying, which is caused by the fact that the input and output signals of the resulting ILC controller have different sampling frequencies. Time-variance is appropriately dealt with in this paper.

The paper is organized as follows. In Section II, the sampled-data ILC problem is formulated. In Section III, the multirate ILC setup is defined and the main theoretical issues regarding multirate systems are presented. In Section IV, the main results regarding the ILC controller design for multirate systems are presented. In Section V, a simulation example, which addresses sampling zeros [8], illustrates the necessity of dealing with intersample behavior in ILC. Finally, in Section VI, concluding remarks are given.

Notation. Throughout, $t \subseteq \mathbb{Z}$ and $t_{c} \subseteq \mathbb{R}$ denotes discrete time and continuous time, respectively. In block diagrams, continuous time signals are represented by solid lines, slowly sampled discrete time signals are represented by dashed lines, and fast sampled discrete time signals are indicated by dotted lines. All systems are assumed to be single-input single-output, finite dimensional, and linear time invariant (LTI). Generalization to the multivariable case is conceptually straightforward. The delay operator $D_{\tau}$ is 
defined by $\left(D_{\tau} f\right)(t)=f(t-\tau)$, where $\tau \in t$. An extended version of the paper is provided in [19].

\section{PRoblem DEFINITION}

In this section, the ILC problem for closed-loop sampleddata systems is defined. The considered setup is depicted in Figure 1 . Here, $y=P u$, where $P$ denotes the continuous time plant. The plant input is given by

$$
\begin{aligned}
u & =\mathcal{H}^{l}\left(w^{l}+C^{d, l} \mathcal{S}^{l} e\right) \\
e & =r-y,
\end{aligned}
$$

where $r$ is the reference signal and $C^{d, l}$ is a discrete time controller operating at a sampling frequency $f^{l}$. In (1), the ideal sampler and zero-order-hold are defined by

$$
\begin{aligned}
\mathcal{S}^{q}: e\left(t_{c}\right) & \mapsto e^{q}(t), e^{q}\left(t_{i}\right)=e\left(t_{i} h^{q}\right) \\
\mathcal{H}^{q}: u^{q}(t) & \mapsto u\left(t_{c}\right), u\left(t_{i} h^{q}+\tau\right)=u^{q}\left(t_{i}\right), \tau=\left[0, h^{q}\right),
\end{aligned}
$$

respectively, where $t_{i} \in t$, sampling frequency $f^{q}=\frac{1}{h^{q}}$, and $h^{q}$ denotes the sampling time. The variable $q$ represents a low or high sampling frequency, e.g., $q=l \Rightarrow h^{q}=h^{l}$. Typically, the sampling time $h^{l}$ of the feedback controller is lower bounded, since a new control signal has to be computed in real-time. The command signal $w^{l}$ is generated by the ILC algorithm. It is assumed that $w^{l}$ operates at the same sampling frequency as the feedback controller, since this is commonly encountered in digital computer implementations. Finally, it is remarked that in (1) and (2), sampled values of the reference signal $r$ could be used, i.e., $\mathcal{S}^{l} r$, since by linearity, see Proposition $6, \mathcal{S}^{l} e=\mathcal{S}^{l} r-\mathcal{S}^{l} y$. However, for the forthcoming sampled-data analysis, it is instructive to consider $r$ as a continuous time signal.

The main problem considered in this paper is given by the norm-optimal sampled-data problem.

Definition 1 (Norm-optimal sampled-data ILC) Given the norm-based criterion $\mathcal{J}_{\mathrm{SD}}\left(w^{l}, e\right)$, the norm-optimal sampled-data ILC problem amounts to determining

$$
w_{\mathrm{SD}^{\star}}^{l}=\arg \min _{w^{l}} \mathcal{J}_{\mathrm{SD}}\left(w^{l}, e\right) .
$$

In the norm-optimal sampled-data ILC problem, an optimal discrete time command signal $w^{l}$ is determined that achieves good continuous time performance $e$, see Figure 1. This implies that the problem involves both continuous time and discrete time signals. This definition of sampled-data systems is consistent with the literature on sampled-data systems, including [1]. In contrast, standard ILC algorithms [3], [4], [6] employ discrete time measurements of the error $e$. In particular, the norm-optimal discrete time ILC problem is given by the following definition.

Definition 2 (Norm-optimal discrete time ILC) Given the norm-based criterion $\mathcal{J}_{\mathrm{DT}}\left(w^{l}, e^{l}\right)$, the norm-optimal discrete ILC problem amounts to determining

$$
w_{\mathrm{DT}^{\star}}^{l}=\arg \min _{w^{l}} \mathcal{J}_{\mathrm{DT}}\left(w^{l}, e^{l}\right) .
$$

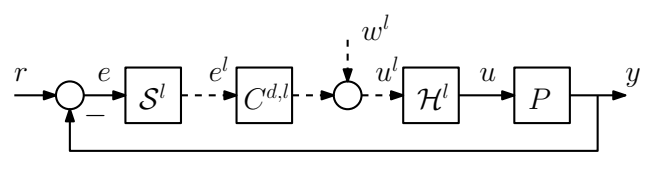

Fig. 1. Closed-loop sampled-data ILC setup.

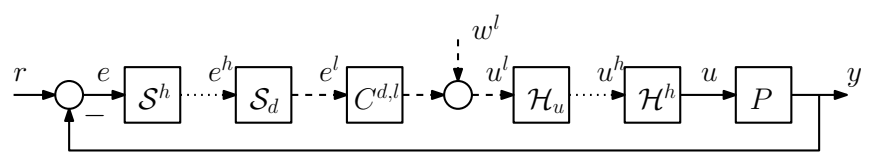

Fig. 2. Closed-loop multirate ILC setup.

In the discrete time ILC criterion $\mathcal{J}_{\mathrm{DT}}\left(w^{l}, e^{l}\right)$, only the atsample response is minimized, whereas the sampled-data criterion $\mathcal{J}_{\mathrm{SD}}\left(w^{l}, e\right)$ includes the intersample response. This implies that discrete time ILC approaches may result in poor intersample behavior, which is quantified by

$$
\mathcal{J}_{\mathrm{SD}}\left(w_{\mathrm{DT}^{\star}}^{l}, e\right) \geq \mathcal{J}_{\mathrm{SD}}\left(w_{\mathrm{SD}^{\star}}^{l}, e\right) .
$$

The gap in (7) depends on the particular system and exogenous signals and can become arbitrarily large, as is illustrated in Section V.

In this paper, the sampled-data ILC problem in Definition 1 is addressed. The sampled-data setup can theoretically be handled using lifted system descriptions, e.g., [1], [20], [21]. However, actual implementation of the resulting ILC controller requires a continuous time measurement of $e$ that is unavailable in a digital computer environment. In the next section, a multirate approximation to the sampled-data ILC problem in Definition 1 is presented to enable a digital computer implementation.

\section{MultiRATE SETUP}

\section{A. Multirate ILC setup}

To enable digital computer implementation of ILC controllers that explicitly address intersample behavior, a multirate approximation of the sampled-data ILC problem, see Definition 1 and Figure 1, is presented. The key idea is that in many applications, it is possible to measure error signals at a higher sampling frequency $f^{h}$ than the frequency $f^{l}$ at which $C^{d, l}$ operates. Indeed, the bound on $f^{l}$ is caused by the fact that in feedback control the new control input has to be computed in real-time. In contrast, although ILC is implemented in real-time, it can exploit the time in between trials for the actual computation of the command signal.

To enable usage of standard tools from multirate signal processing [22], the following assumption is imposed.

Assumption 3 Let the sampling frequencies $f^{l}$ and $f^{h}$ be related by

$$
f^{h}=F f^{l}, \quad 1<F \in \mathbb{Z} .
$$

The multirate ILC setup is depicted in Figure 2. Herein, $\mathcal{S}^{h}$ and $\mathcal{H}^{h}$ are defined in (3) and (4), respectively, where $q=h$, i.e., a high sampling frequency $f^{h}$ is assumed. The downsampling operator $\mathcal{S}_{d}$ is defined by

$$
\mathcal{S}_{d}: e^{h}(t) \mapsto e^{l}(t), e^{l}\left(t_{i}\right)=e^{h}\left(F t_{i}\right), \quad t_{i} \in t .
$$


In addition, the multirate zero-order-hold $\mathcal{H}_{u}$ is defined as [2]

$$
\mathcal{H}_{u}=\mathcal{I}^{F}(z) \mathcal{S}_{u}
$$

where the upsampler $\mathcal{S}_{u}$ and zero-order-hold interpolator $\mathcal{I}^{F}(z)$ are given by

$$
\mathcal{S}_{u}: u^{l}(t) \mapsto \tilde{u}^{h}(t), \tilde{u}^{h}\left(t_{i}\right):= \begin{cases}u^{l}\left(\frac{t_{i}}{F}\right) & \text { for } t_{i} \in t, \frac{t_{i}}{F} \in \mathbb{Z} \\ 0 & \text { for } t_{i} \in t, \frac{t_{i}}{F} \notin \mathbb{Z} .\end{cases}
$$

$$
\mathcal{I}^{F}(z)=\sum_{f=0}^{F-1} z^{-f}
$$

The multirate setup in Figure 2 leads to the following norm-optimal multirate ILC problem.

Definition 4 (Norm-optimal multirate ILC) Given the norm-based criterion $\mathcal{J}_{\mathrm{MR}}\left(w^{l}, e^{h}\right)$, the norm-optimal multirate ILC problem amounts to determining

$$
w_{\mathrm{MR}^{\star}}^{l}=\arg \min _{w^{l}} \mathcal{J}_{\mathrm{MR}}\left(w^{l}, e^{h}\right) .
$$

The norm-optimal multirate ILC problem is a sensible approximation of the sampled-data ILC problem, since under appropriate technical conditions [23]

$$
\mathcal{J}_{\mathrm{SD}}\left(w^{l}, e\right)-\mathcal{J}_{\mathrm{MR}}\left(w^{l}, e^{h}\right) \rightarrow 0 \text { for } h^{h} \rightarrow 0 .
$$

In practice, $F$ and hence $h^{h}$, see (8), are upper bounded. In the zero-order-hold case, a low $F$, e.g., $2 \leq F \leq 5$ typically suffices due to the low-pass character of the interpolator [2], [10]. In the next section, the multirate setup in Figure 2 is analyzed in detail.

\section{B. Multirate analysis}

In this section, the multirate setup in Figure 2 is analyzed to enable proper formulation of the multirate system description used for ILC. Initially, admissible feedback controllers and sampling frequencies are discussed. Subsequently, linearity and time-variance of the multirate ILC setup are investigated.

Throughout, it is assumed that the feedback system in Figure 1 is well-posed and internally stable [24]. A nonpathological sampling frequency is assumed, which is formalized in the following assumption [25].

Assumption 5 Let $(A, B, C, D)$ be a minimal state space realization of $P$. Then, it is assumed that $A$ does not have two eigenvalues $\lambda_{p}(A)=\sigma_{p}+j \omega_{p}$ and $\lambda_{q}(A)=\sigma_{q}+j \omega_{q}$, $p \neq q$ that satisfy

$$
\left\{\lambda_{p}, \lambda_{q} \mid \sigma_{p}=\sigma_{q} \text { and } \omega_{p}=\omega_{q}+r 2 \pi f^{l}, r \in \mathbb{Z} \backslash\{0\}\right\} .
$$

Assumption 5 ensures sampling preserves observability and controllability. Hence, there cannot be any unstable modes in the intersample behavior.

Next, linearity and time-variance of the multirate system are investigated. The following proposition reveals that sampling and hold operators are linear.
Proposition 6 The operators $\mathcal{S}^{q}, \mathcal{H}^{q}, \mathcal{S}_{d}$, and $\mathcal{H}_{u}$, in (3), (4), (9), and (10), respectively, are linear.

The proof of Proposition 6 follows directly from the definition of linearity [24].

To analyze time-variance, the notion of periodically timevarying operators is useful.

Definition 7 [1], [20] An operator $G$ is periodically timevarying with period $h$ if it commutes with the delay operator $D_{h}$, i.e., $D_{h} G=G D_{h}$, where $h \in t$.

If Definition 7 does not apply, then the system is considered time-varying. Recall that time-invariance is a special case of periodical time variance.

Definition 8 An operator $G$ is time-invariant if it commutes with $D_{h}$ for all $h \in t$.

The following results reveal that linearity is preserved in multirate systems, yet time-invariance may be lost, i.e., multirate systems can become linear periodically time-varying (LPTV).

Proposition 9 Consider the sampler $\mathcal{S}^{q}$ and zero-order-hold $\mathcal{H}^{q}$. Then,

(a) $\mathcal{S}^{q} \mathcal{H}^{q}$ is an LTI operator

(b) $\mathcal{H}^{q} \mathcal{S}^{q}$ is an LPTV operator with period $h^{q}$.

Proof: (a): (3) and (4) yield $\mathcal{S}^{q} \mathcal{H}^{q}=I$ that is LTI. (b): follows from (3) and (4) and evaluating the delay operator $D_{\tau}$ for different values of $\tau$.

The following results are required for the manipulation of multirate systems in the next sections.

Proposition 10 Consider the setup in Figure 2 and let $(A, B, C, D)$ be defined as in Assumption 5. Then, the following properties hold:

(a) $\mathcal{S}^{l}=\mathcal{S}_{d} \mathcal{S}^{h}$

(b) $\mathcal{H}^{l}=\mathcal{H}^{h} \mathcal{H}_{u}$

(c) $P^{d, h}=\mathcal{S}^{h} P \mathcal{H}^{h}$ has state-space realization $\left(A^{h}, B^{h}, C, D\right)$

(d) $P^{d, l}=\mathcal{S}^{l} P \mathcal{H}^{l}=\mathcal{S}^{d} P^{d, h} \mathcal{H}^{u}$ has state-space realization $\left(A^{l}, B^{l}, C, D\right)$

(e) $P^{d, h}$ and $P^{d, l}$ are LTI and minimal, where $A^{h}=e^{A h^{h}}, B^{h}=\int_{0}^{h^{h}} e^{A \tau} d \tau B$, and $A^{l}$ and $B^{l}$ follow from $\left[\begin{array}{cc}A^{l} & B^{l} \\ \star & \star\end{array}\right]=\left[\begin{array}{cc}A^{h} & B^{h} \\ 0 & I\end{array}\right]^{F}$, where $\star$ is not used in further computations.

Proof: (a), (b): follows from (3), (9) and (4), (10), respectively. (c): follows by integrating differential equations. (d): follows by successive substitution in the difference equations. (e): LTI follows similarly as in Proposition 9, minimality follows if Assumption 5 is included in (c) and (d).

The novelty of Proposition 10 is that the sampling frequency of $P^{d, h}$ can be reduced to obtain $P^{d, l}$, which will be exploited in Section IV. 
Propositions 9 and 10 (e) also have important implications in the analysis of multirate and sampled-data systems. To illustrate this, consider an operator that consists of a series connection of LTI operators and samplers and holds. If the input, the output, or both the input and output are signals operating at a higher sampling frequency than any of the linear operators in between, then the resulting system is LPTV. This implies that standard transfer functions do not apply [24] and sinusoids are no longer eigenfunctions of such operators. In contrast, if the input and output are both signals at the lowest sampling frequency $f^{l}$ compared to any of the linear operators in between, then the resulting operator is LTI and operates at a sampling frequency $f^{l}$. The LTI nature of these systems is essential in standard discrete time operations on signals and systems based on LTI assumptions.

\section{Multirate expressions for ILC}

ILC algoritms require a model that represents the operator between the reference $r$, command input $w^{l}$, and sampled versions of $e$. Depending on the approach, either discrete time ILC or multirate ILC, the sampling frequency of $e$ is $f^{l}$ or $f^{h}$, respectively. In this section, these operators are derived and analyzed using the results in Section III-B. Specifically, the operators $w^{l} \mapsto e^{l}$ and $w^{l} \mapsto e^{h}$ are required for the discrete time ILC and multirate ILC problems, respectively.

Proposition 11 Consider the closed-loop system of Figure 2. Then, the operators mapping $w^{l}$ and $r$ to $e^{l}$ and $e^{h}$ are given by

$$
\begin{aligned}
e^{l}= & \left(I-P^{d, l}\left(I+C^{d, l} P^{d, l}\right)^{-1} C^{d, l}\right) \mathcal{S}^{l} r-J_{\mathrm{DT}} w^{l} \\
J_{\mathrm{DT}}= & P^{d, l}\left(I+C^{d, l} P^{d, l}\right)^{-1} \\
e^{h}= & \left(I-P^{d, h} \mathcal{H}_{u}\left(I+C^{d, l} P^{d, l}\right)^{-1} C^{d, l} \mathcal{S}_{d}\right) \mathcal{S}^{h} r \\
& \quad-J_{\mathrm{MR}} w^{l} \\
J_{\mathrm{MR}}= & P^{d, h} \mathcal{H}_{u}\left(I+C^{d, l} P^{d, l}\right)^{-1} .
\end{aligned}
$$

In addition, the mapping $-J_{\mathrm{DT}}: w^{l} \mapsto e^{l}$ is LTI, whereas the mapping $-J_{\mathrm{MR}}: w^{l} \mapsto e^{h}$ is time-varying.

Proof: Equations (16) and (18) result from the interconnection structure in Figure 2 and Proposition 10. The fact that $w^{l} \mapsto e^{l}$ is LTI follows from Proposition 10 (f). Timevariance of $w^{l} \mapsto e^{h}$ follows from Definitions 7 and 8 .

Time-variance of $w^{l} \mapsto e^{h}$ is best interpreted by the fact that the delay operator applied to $w^{l}$ corresponds to time steps $h^{l}$, whereas the delay operator applied to $e^{h}$ corresponds to time steps $h^{h}$. Additionally, if a fictitious signal satisfying $w^{l}=\mathcal{S}_{d} w^{h}$ is introduced, then the mapping $w^{h} \mapsto e^{h}$ is LPTV, see Proposition 9 (b).

The main consequence of Proposition 11 is that discrete time ILC can resort to standard LTI design techniques. The mapping $w^{l} \mapsto e^{h}$, as required in multirate ILC, is time-varying, hence transfer functions in the usual sense do not apply and standard LTI design techniques cannot be employed. In the next section, a general framework for multirate ILC is proposed that deals with the time-varying nature of the mapping $w^{l} \mapsto e^{h}$ and provides a solution to the multirate ILC problem in Definition 4.

\section{Multirate ILC}

In this section, the solution to the multirate ILC problem is presented. In Section IV-A, appropriate finite time system descriptions of the required time-varying operators are presented. Then, in Section IV-B, the norm-optimal ILC controller is presented. Finally, design aspects and convergence results are discussed in Section IV-C.

\section{A. Finite time system descriptions}

Consider the system $P^{d, l}$ with Markov parameters $m_{t_{i}}^{l}$, operating over a finite time interval $t_{i} \in\left[0, N^{l}-1\right] \subseteq t$, where the state of the system is reset to zero after each trial. Then, the input-output behavior is represented by its convolution matrix [26], [27]:

$$
\underline{y}^{l}=\underline{P}^{d, l} \underline{u}^{l}, \quad \underline{P}^{d, l}=\left[\begin{array}{ccc}
m_{0}^{l} & & 0 \\
\vdots & \ddots & \\
m_{N^{l}-1}^{l} & \cdots & m_{0}^{l}
\end{array}\right] .
$$

For causal SISO LTI systems, $\underline{P}^{d, l} \in \mathbb{R}^{N^{l} \times N^{l}}$ is a square lower triangular Toeplitz matrix that maps input vector $\underline{u}^{l} \in$ $\mathbb{R}^{N^{l}}$ to output vector $\underline{y}^{l} \in \mathbb{R}^{N^{l}}$. The signals $\underline{u}^{l}$ and $\underline{y}^{l}$ are obtained by stacking the corresponding time signals for time interval $t_{i} \in\left[0, N^{l}-1\right]$ in vectors.

Similar to $P^{d, l}$, the finite time representation of $P^{d, h}$ with Markov parameters $m_{t_{i}}^{h}$ for $t_{i} \in\left[0, F\left(N^{l}-1\right)\right] \subseteq t$, is given by $\underline{P}^{d, h}$. In this case, system $\underline{P}^{d, h} \in \mathbb{R}^{F N^{l} \times F N^{l}}$ maps input $\underline{u}^{h} \in \mathbb{R}^{F N^{l}}$ to output $\underline{y}^{h} \in \mathbb{R}^{\bar{F} N^{l}}$.

Next to finite time LTI system descriptions, the presented framework can be used to formulate finite time expressions for the downsampler $\mathcal{S}_{d}$ and multirate zero-order-hold $\mathcal{H}_{u}$ using block Toeplitz matrices, i.e.,

$$
\begin{aligned}
& \underline{\mathcal{S}}_{d}=I_{F} \otimes\left[\begin{array}{ll}
1 & 0_{F-1}
\end{array}\right], \quad \underline{\mathcal{S}}_{d} \in \mathbb{R}^{N^{l} \times F N^{l}} \\
& \underline{\mathcal{H}}_{u}=I_{F} \otimes \underline{\mathbf{1}}_{F}, \quad \underline{\mathcal{H}}_{u} \in \mathbb{R}^{F N^{l} \times N^{l}},
\end{aligned}
$$

respectively, where $\underline{\mathbf{1}}_{F}:=\left[\begin{array}{lll}1 & \cdots & 1\end{array}\right]^{T} \in \mathbb{R}^{F}, I_{F} \in \mathbb{R}^{F \times F}$ denotes the identity matrix, and $\otimes$ denotes the Kronecker product [24]. With $\underline{P}^{d, l}$ equal to $\underline{\mathcal{S}}_{d} \underline{P}^{h} \underline{\mathcal{H}}_{u}$, in general $m_{t_{i}}^{l} \neq$ $m_{F t_{i}}^{h}$. In addition, $\underline{\mathcal{S}}_{d}$ and $\underline{\mathcal{H}}_{u}$ are non-square block Toeplitz matrices that correspond to sample rate conversions, and hence to time-varying behavior.

\section{B. Norm-optimal multirate ILC}

To determine the ILC controllers, the finite time mapping between the command signal and error in Proposition 11 is required, see also [17].

Proposition 12 Consider the interconnection structure in Figure 2. Then, the finite time mappings $-\underline{J}_{\mathrm{DT}}: \underline{w}^{l} \mapsto \underline{e}^{l}$ and $-\underline{J}_{\mathrm{MR}}: \underline{w}^{l} \mapsto \underline{e}^{h}$, corresponding to (17) and (19), respectively, are given by

$$
\begin{aligned}
& \underline{J}_{\mathrm{DT}}=\underline{P}^{d, l}\left(I_{N^{l}}+\underline{C}^{d, l} \underline{P}^{d, l}\right)^{-1} \in \mathbb{R}^{N^{l} \times N^{l}} \\
& \underline{J}_{\mathrm{MR}}=\underline{P}^{d, h} \underline{\mathcal{H}}_{u}\left(I_{N^{l}}+\underline{C}^{d, l} \underline{P}^{d, l}\right)^{-1} \in \mathbb{R}^{F N^{l} \times N^{l}} .
\end{aligned}
$$

In addition, $\underline{J}_{\mathrm{DT}}=\underline{\mathcal{S}}_{d} \underline{J}_{\mathrm{MR}}$. 
With the relevant systems and signals for ILC defined, the norm-optimal ILC controller can be designed. In general, the objective in ILC control design is to minimize the error during trial $k+1, k \in \mathbb{Z}_{+}$in an appropriate norm by determining a suitable command signal. In norm-optimal ILC, additional criteria are included in the objective to bound the command amplitude and the change in amplitude of the command signal from trial $k$ to trial $k+1$. Specifically, the criterion for determining the command input $\underline{w}^{l}$ in trial $k+1$ is given by

$$
\begin{gathered}
\mathcal{J}<k+1>=\underline{e}^{q T}<k+1>\underline{W}_{e} \underline{e}_{<k+1>}^{q}+\underline{w}^{l T}<k+1>\underline{W}_{w} \underline{w}^{l}<k+1> \\
+\left(\underline{w}^{l}<k+1>-\underline{w}^{l}<k>\right)^{T} \underline{W}_{\Delta w}\left(\underline{w}^{l}<k+1>-\underline{w}^{l}<k>\right),
\end{gathered}
$$

where $q=l$ for discrete time ILC and $q=h$ in multirate ILC. In addition, $\underline{W}_{e}, \underline{W}_{w}$, and $\underline{W}_{\Delta w}$ are weighting matrices of appropriate sizes.

The resulting optimal multirate ILC controller is the main result of this section and is given by the following proposition.

Proposition 13 Given a multirate system $-\underline{J}_{\mathrm{MR}}: \underline{w}^{l} \mapsto \underline{e}^{h}$ and criterion (25). Then, the norm-optimal multirate ILC controller $\left(\underline{Q}_{\mathrm{MR}}, \underline{L}_{\mathrm{MR}}\right)$ is given by

$$
\begin{aligned}
\underline{w}^{l}<k+1> & =\underline{Q}_{\mathrm{MR}} \underline{w}^{l}<k> \\
\underline{Q}_{\mathrm{MR}} & =\left(\underline{L}_{\mathrm{MR}} \underline{\underline{U}}_{\mathrm{MR}>}^{q} \underline{W}_{e} \underline{J}_{\mathrm{MR}}+\underline{W}_{w}+\underline{W}_{\Delta w}\right)^{-1} \\
& \cdot\left(\underline{J}_{\mathrm{MR}}^{T} \underline{W}_{e} \underline{J}_{\mathrm{MR}}+\underline{W}_{\Delta w}\right) \\
\underline{L}_{\mathrm{MR}}= & \left(\underline{J}_{\mathrm{MR}}^{T} \underline{W}_{e} \underline{J}_{\mathrm{MR}}+\underline{W}_{w}+\underline{W}_{\Delta w}\right)^{-1} \underline{J}_{\mathrm{MR}}^{T} \underline{W}_{e} .
\end{aligned}
$$

The solution of the norm-optimal multirate ILC problem seems to be novel. Specifically, the multirate solution involves non-square matrices, enabling the multirate ILC controller to map fastly sampled error signals, i.e., $e^{h}$, into slowly sampled command signals, i.e., $w^{l}$. In contrast, the discrete time ILC problem results in square matrices, requiring an identical sampling frequency of the error signal, i.e., $e^{l}$, and the command signal $w^{l}$. In fact, the discrete time result can directly be recovered using Proposition 12, since the discrete time problem is a special case of the ILC problem for $F=1$. The proof of Proposition 13 follows along similar lines as the discrete time norm-optimal ILC problem, see [28], [27], [6].

\section{Design aspects}

To apply the multirate ILC approach in the previous sections, a model of the system $\underline{J}_{\mathrm{MR}}$ is required. Given $P^{d, h}$ and $C^{d, l}, \underline{J}_{\mathrm{MR}}$ can be computed using the results of Proposition 10 and Proposition 12.

In (25), the weighting matrices $\underline{W}_{e}, \underline{W}_{w}$, and $\underline{W}_{\Delta w}$ are used to emphasize the relative importance of the different criteria. Requiring that all matrices are positive definite is a sufficient condition for a well-posed optimization problem and guaranteed monotonic convergence in $w^{l}$ [28], i.e.,

$$
\left\|\underline{w}_{<k+1>}^{l}-\underline{w}_{<\infty>}^{l}\right\|_{2}<\left\|\underline{w}_{<k>}^{l}-\underline{w}_{<\infty>}^{l}\right\|_{2},
$$

where $k \in \mathbb{Z}_{+}$and $\underline{w}_{<\infty>}^{l}=\lim _{k \rightarrow \infty} \underline{w}_{<k>}^{l}$. Often, the weighting filters are selected as

$$
\underline{W}_{e}=r_{e} I, \quad \underline{W}_{w}=r_{w} I, \quad \underline{W}_{\Delta w}=r_{\Delta w} I,
$$

where $r_{e}, r_{w}, r_{\Delta w} \in \mathbb{R}_{+}$. In case of (30), $r_{\Delta w}$ affects the convergence speed, yet not the converged error for $k \rightarrow \infty$. Selecting a large $r_{\Delta w}$ is useful to attenuate the influence of trial-varying exogenous signals, including measurement noise. The parameters $r_{e}$ and $r_{w}$ can be used to weigh the tracking error and the control effort. A large $r_{e}$ relative to $r_{w}$ results in a small tracking error, whereas a large $r_{w}$ results in a smaller control effort and improved robustness against model uncertainty [29].

Finally, it is noted that the discrete time criterion $\mathcal{J}_{\mathrm{DT}}$ and multirate criterion $\mathcal{J}_{\mathrm{MR}}$ cannot be compared directly, since $e^{l}$ and $e^{h}$ are defined in different function spaces. Hence, suitable modifications should be made for comparing $\mathcal{J}_{\mathrm{DT}}$ and $\mathcal{J}_{\mathrm{MR}}$. Specifically, by considering $\mathcal{H}_{u} e^{l}$, the normoptimal discrete time ILC problem in Definition 2 can be cast in the criterion in Definition 4. In this case, the weighting matrix $W_{e}$ in (30), corresponding to the criterion $\mathcal{J}_{\mathrm{DT}}$, should be scaled appropriately by $\frac{r_{e}}{F}$.

\section{EXAMPLE}

In this section, the ILC approach of the previous sections is applied to a simulation model of a motion system.

\section{A. Setup}

The considered system is a positioning system, which is represented by the differential equation

$$
m \ddot{x}=k u, \quad y=\left[\begin{array}{ll}
x & \dot{x}
\end{array}\right]^{T},
$$

where the mass $m$ and motor constant $k$ are normalized, i.e., $k=m=1$. In addition, for the sake of the example, it is assumed that both the position and velocity are measured. The system (31) is unstable, hence a stabilizing discrete time feedback controller $C^{d, l}$, which is a lead-lag controller that again for the sake of the example only uses the position measurement, is implemented with a sampling time $h^{l}=$ 0.01 s, i.e., $f^{l}=\frac{1}{h^{l}}=100 \mathrm{~Hz}$. Specifically,

$$
P=\left[\begin{array}{c}
P_{11} \\
P_{21}
\end{array}\right]=\left[\begin{array}{c}
\frac{1}{s^{2}} \\
\frac{1}{s}
\end{array}\right], \quad C^{d, l}=\left[\frac{126 z-123.6}{z-0.8282} \quad 0\right] .
$$

The sampling time of the feedback controller is restricted, since at each sample instant a new control input has to be computed. However, it is possible to record measurement data with a sampling time of $h^{h}=0.0025$ s, hence $F=4$ and the sampling frequency $f^{h}=\frac{1}{h^{h}}=400 \mathrm{~Hz}$. The measurement data recorded with a sampling time $h^{h}$ is available for the ILC algorithm. The ILC setup and feedback interconnection are depicted in Figure 2, where

$$
e=\left[\begin{array}{l}
e_{1} \\
e_{2}
\end{array}\right]=\left[\begin{array}{l}
r-x \\
\dot{r}-\dot{x}
\end{array}\right] .
$$

In the sequel, only scalar ILC is considered, i.e., sampled measurements of either $e_{1}$ or $e_{2}$ are used as input for the ILC algorithm. In all cases, the considered exogenous variables are trial invariant, except for the command signal 
TABLE I

EXAMPLE 1: RESULTING CRITERION VALUE.

\begin{tabular}{l|cc} 
& $\mathcal{J}_{\mathrm{DT}}\left(w^{l}, e^{l}\right)$ & $\mathcal{J}_{\mathrm{MR}}\left(w^{l}, e^{h}\right)$ \\
\hline Initial $k=0$ & 52.0 & 52.0 \\
Discrete time ILC $k=10$ & 0 & 10.3 \\
Multirate ILC $k=10$ & 0.7 & 0.3
\end{tabular}

$w^{l}$. Throughout this section, $r_{\Delta w^{l}}=10^{-12}$. In addition, the parameter $r_{e}=1$ in $\mathcal{J}_{\mathrm{MR}}$ and $r_{e}=4$ in $\mathcal{J}_{\mathrm{DT}}$, see Section IVC. To compare the presented multirate ILC approach with standard discrete time ILC, both multirate and discrete time ILC are applied, which is indicated by the subscripts DT and MR, respectively. For instance, the resulting error at sampling frequency $f^{l}$ after 10 iterations of the discrete time ILC algorithm is denoted by $e_{\mathrm{DT}<10>}^{l}$.

\section{B. Example 1}

In this example, the position error $e_{1}$ is used in both the discrete time and multirate ILC algorithms, where $r_{w^{l}}=0$ in (30). In the first trial, i.e., $k=0$, the command input $w_{<0>}^{l}=0$. The corresponding errors $e_{1,<0>}^{l}$ and $e_{1,<0>}^{h}$, measured at sampling frequencies $f^{l}$ and $f^{h}$, respectively, are depicted in Figure 3 (a).

Firstly, the discrete time ILC algorithm is applied. The error after 10 iterations is depicted in Figure 3 (b), both at sampling frequencies $f^{l}$ and $f^{h}$. The error $e_{\mathrm{DT}<k>}^{h}$ is not used by the discrete time ILC algorithm, only for analysis of the results. The ILC algorithm achieves zero tracking error $e_{\mathrm{DT}<10>}^{l}$ after 10 iterations. However, this zero tracking error is at the expense of a poor intersample behavior. These results are confirmed by evaluating the criteria $\mathcal{J}_{\mathrm{DT}}$ and $\mathcal{J}_{\mathrm{MR}}$, see Table I. The poor intersample behavior cannot be observed from $e^{l}$ and $\mathcal{J}_{\mathrm{DT}}$, hence such criteria are not suitable for analyzing the performance of sampled-data systems.

Secondly, the multirate ILC algorithm is applied, with $e_{\mathrm{MR}<k>}^{h}$ the input to the algorithm. Though the multirate ILC algorithm results after 10 iterations in a larger error at the sampling frequency $f^{l}$, the multirate ILC algorithm results in improved intersample behavior compared to discrete time ILC, see Figure 3 (c) and Table I.

The poor intersample behavior in the discrete time ILC case can be attributed to the cancellation of a sampling zero [8]. Discretization of the system in (32) yields

$$
P_{11}^{d, l}=\frac{h^{l}}{2}(z+1) /(z-1)^{2},
$$

where a sampling zero at $z=1$ appears due to the relative degree 2 of the system $P_{11}$. The discrete time ILC algorithm in fact cancels this sampling zero, resulting in the poor intersample behavior. The location of the sampling zero in (34) is invariant under a changing sampling frequency, hence modifying the sampling frequency in the discrete time approach does not change the results.

\section{Example 2}

In Section V-B, it is concluded that neglecting sampling zeros in discrete time ILC can result in poor intersample behavior. By analyzing Figure 3, it can be concluded that the
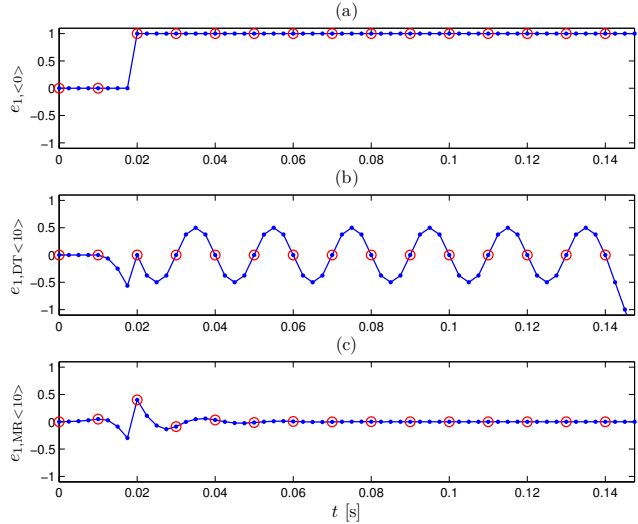

Fig. 3. Example 1: Comparison of position errors $e_{1}$ at sampling frequency $f^{h}$ (dots) and $f^{l}$ (circles), $r_{w^{l}}=0$, (a): initial, (b): after 10 trials discrete time ILC, (c): after 10 trials multirate ILC.

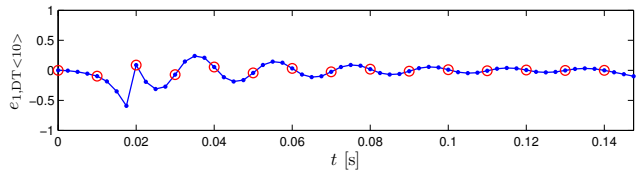

Fig. 4. Example 2: Comparison of position errors $e_{1}$ at sampling frequency $f^{h}$ (dots) and $f^{l}$ (circles), $r_{w^{l}}=10^{-11}$ : after 10 trials discrete time ILC.

intersample oscillation requires a nonzero command signal $w^{l}$. Hence, an ad hoc solution to resolve the intersample behavior issue in discrete time ILC in case of sampled zeros is to include input weighting in criterion (25). In Figure 4 the results are depicted for $r_{w^{l}}=10^{-11}$. Compared to Example 1 in Section V-B, inclusion of input weighting in ILC results in a significantly lower error at the higher sampling frequency $f^{h}$ at the expense of a slightly larger error at the low sampling frequency $f^{l}$, and hence better intersample behavior. Still, the multirate ILC algorithm outperforms the discrete time algorithm if the error is considered at sampling frequency $f^{h}$, see Table I.

\section{Example 3}

In Section V-B and V-C, it was shown that standard ILC may result in cancellation of sampling zeros, which in turn result in poor intersample behavior. In this section, it is shown that the poor intersample behavior can also be resolved by reducing the relative degree of the system such that sampling zeros do not appear. To achieve this, the velocity error $e_{2}$, see (33), is considered in the ILC approach. The initial error $e_{2}$ at trial $k=0$ is depicted in Figure 5 (a). The resulting errors after 10 trials of discrete time and multirate ILC with $r_{w^{l}}=0$ are depicted in Figure 5 (b) and Figure 5 (c), respectively. The corresponding criteria $\mathcal{J}_{\mathrm{DT}}$ and $\mathcal{J}_{\mathrm{MR}}$ are presented in Table II. Clearly, the discrete time ILC algorithm results in zero tracking error at sampling frequency $f^{l}$, whereas the intersample behavior remains acceptable. From the results in Table II, it is concluded that the multirate ILC approach results in a better balanced tradeoff between the error at sampling frequency $f^{l}$ and intersample behavior, evaluated at a sampling frequency $f^{h}$. 

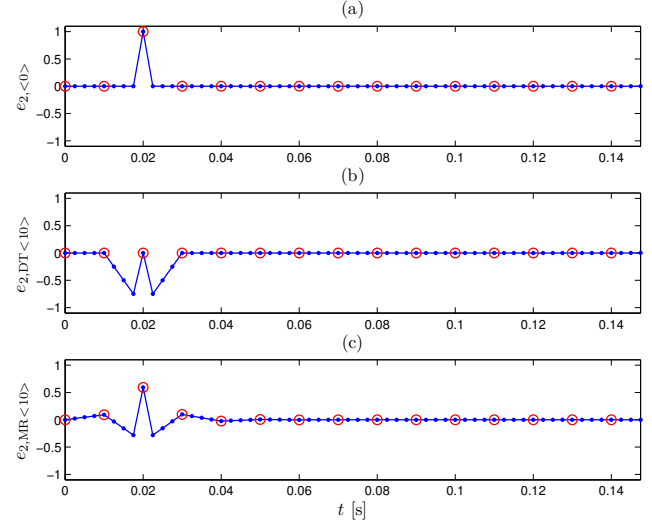

Fig. 5. Example 3: Comparison of errors at sampling frequency $f^{h}$ (dots) and $f_{s}$ (circles), $r_{w^{l}}=0$, (a): initial error, (b): error after 10 trials discrete time ILC, (c): error after 10 trials multirate ILC.

TABLE II

EXAMPLE 1.3: RESULTING CRITERION VALUE.

\begin{tabular}{l|cc} 
& $\mathcal{J}_{\mathrm{DT}}\left(w^{l}, e^{l}\right)$ & $\mathcal{J}_{\mathrm{MR}}\left(w^{l}, e^{h}\right)$ \\
\hline Initial $k=0$ & 4.0 & 1.0 \\
Discrete time ILC $k=10$ & 0 & 1.8 \\
Multirate ILC $k=10$ & 1.5 & 0.6
\end{tabular}

\section{CONCLUSIONS}

A novel ILC framework for sampled-data systems aiming at high continuous time performance is presented. The presented approach extends common, discrete time ILC approaches by explicitly addressing the intersample behavior in the learning algorithm. A multirate approach is pursued to enable actual implementation in a digital computer environment. In the limiting case, the multirate problem converges to the sampled-data problem. In practice, a small sampling frequency ratio $F$ is sufficient to approximate the sampled-data ILC problem due to the low-pass characteristic of the zero-order-hold interpolator. In addition, key issues in sampled-data and multirate control, including the timevarying nature of the multirate ILC setup, have been dealt with appropriately in the norm-optimal ILC framework.

In a realistic simulation example, it is shown that the proposed multirate ILC approach outperforms discrete time ILC. Specifically, discrete time ILC results in poor intersample behavior in case of sampling zeros. Multirate ILC deals with both these phenomena appropriately by balancing the error at the low sampling frequency and the intersample behavior. The presented approach also handles the case of aliased disturbances [2] and aliased poles [10].

Extensions of the framework include a noninteger sampling frequency ratio $F$, however, in this case Proposition 10 and the block Toeplitz results in Section IV do not apply directly.

\section{REFERENCES}

[1] T. Chen and B. Francis, Optimal Sampled-Data Control Systems. London, UK: Springer, 1995.

[2] T. Oomen, M. van de Wal, and O. Bosgra, "Design framework for high-performance optimal sampled-data control with application to a wafer stage," Int. J. Contr., vol. 80, no. 6, pp. 919 - 934, 2007.
[3] K. L. Moore, "Iterative learning control - an expository overview," Appl. Comp. Contr. Sign. Proc. Circ., vol. 1, pp. 151-214, 1999.

[4] D. A. Bristow, M. Tharayil, and A. G. Alleyne, "A survey of iterative learning control: A learning-based method for high-performance tracking control," Contr. Syst. Mag., vol. 26, no. 3, pp. 96-114, 2006.

[5] Z. Bien and J.-X. Xu, Iterative Learning Control: Analysis, Design, Integration and Applications. Norwell, MA, USA: Kluwer Academic Publishing, 1998.

[6] D. Gorinevsky, "Loop shaping for iterative control of batch processes," Contr. Syst. Mag., vol. 22, no. 6, pp. 55-65, 2002.

[7] K. J. Åström and B. Wittenmark, Computed-Controlled Systems: Theory and Design, 2nd ed. Englewood Cliffs, NJ, USA: PrenticeHall, 1990.

[8] K. J. Åström, P. Hagander, and J. Sternby, "Zeros of sampled systems," Automatica, vol. 20, no. 1, pp. 31-38, 1984.

[9] S. R. Weller and R. H. Middleton, "On the role of sampling zeros in robust sampled-data control design," in Proc. 37th Conf. Dec. Contr., Tampa, FL, USA, 1998, pp. 325-330.

[10] T. Oomen, M. van de Wal, and O. Bosgra, "Aliasing of resonance phenomena in sampled-data control design: Hazards, modeling, and a solution," in Proc. 2007 Americ. Contr. Conf., New York, NY, USA, 2007, pp. $2881-2886$.

[11] H.-K. Sung and S. Hara, "Properties of sensitivity and complementary sensitivity functions in single-input single-output digital control systems," Int. J. Contr., vol. 48, no. 6, pp. 2429-2439, 1988.

[12] R. W. Longman and C.-P. Lo, "Generalized holds, ripple attenuation, and tracking additional outputs in learning control," J. Guid., Contr., Dyn., vol. 20, no. 6, pp. 1207-1214, 1997.

[13] P. A. LeVoci and R. W. Longman, "Intersample error in discrete time learning and repetitive control," in Proc. AIAA/AAS Astrodynamics Specialist Conf. and Exhibit, Providence, RI, USA, 2004, pp. 1-24.

[14] S. Hara, M. Tetsuka, and R. Kondo, "Ripple attenuation in digital repetitive control systems," in Proc. 29th Conf. Dec. Contr., Honolulu, HI, USA, 1990, pp. 1679-1684.

[15] A. Langari and B. A. Francis, "Sampled-data repetitive control systems," in Proc. 13th Americ. Contr. Conf., Baltimore, MD, USA, 1994, pp. 3234-3235.

[16] H. Ishii and Y. Yamamoto, "Periodic compensation for sampled-data $\mathcal{H}_{\infty}$ repetitive control," in Proc. 37th Conf. Dec. Contr., Tampa, FL, USA, 1998, pp. 331-336.

[17] J. van de Wijdeven and O. Bosgra, "Residual vibration suppression using Hankel iterative learning control," Int. J. Rob. Nonlin. Contr., vol. 18, no. 10, pp. 1034-1051, 2008.

[18] B. Zhang, D. Wang, Y. Ye, Y. Wang, and K. Zhou, "Two-mode ILC with pseudo-downsampled learning in high frequency range," Int. J. Contr., vol. 80, no. 3, pp. 349-362, 2007.

[19] T. Oomen, J. van de Wijdeven, and O. Bosgra, In preparation for journal publication.

[20] B. Bamieh, J. B. Pearson, B. A. Francis, and A. Tannenbaum, "A lifting technique for linear periodic systems with applications to sampled-data control," Syst. Contr. Lett., vol. 17, no. 2, pp. 79-88, 1991.

[21] Y. Yamamoto, "A function space approach to sampled data control systems and tracking problems," IEEE Trans. Automat. Contr., vol. 39, no. 4, pp. 703-713, 1994.

[22] P. P. Vaidyanathan, Multirate Systems and Filter Banks. Englewood Cliffs, NJ, USA: Prentice Hall, 1993.

[23] Y. Kannai and G. Weiss, "Approximating signals by fast impulse sampling," Math. Contr. Sign. Syst., vol. 6, no. 2, pp. 166-179, 1993.

[24] K. Zhou, J. C. Doyle, and K. Glover, Robust and Optimal Control. Upper Saddle River, NJ, USA: Prentice Hall, 1996.

[25] R. E. Kalman, Y. C. Ho, and K. S. Narendra, Contributions to Differential Equations. New York, NY, USA: Interscience, 1963, vol. 1, no. 2, ch. Controllability of Linear Dynamical Systems, pp. $189-213$.

[26] M. Phan and R. W. Longman, "A mathematical theory of learning control for linear discrete multivariable systems," in Proc. AIAA/AAS Astrodynamics Conf., Minneapolis, MN, USA, 1988, pp. 740-746.

[27] J. A. Frueh and M. Q. Phan, "Linear quadratic optimal learning control (LQL)," Int. J. Contr., vol. 73, no. 10, pp. 832-839, 2000.

[28] S. Gunnarsson and M. Norrlö, "On the design of ILC algorithms using optimization," Automatica, vol. 37, pp. 2011-2016, 2001.

[29] T. Donkers, J. van de Wijdeven, and O. Bosgra, "Robustness against model uncertainties of norm optimal iterative learning control," in Proc. 2008 Americ. Contr. Conf., Seattle, WA, USA, 2008, pp. 45614566. 